\title{
Percepção de Dor Aguda em Pacientes com Transtorno de Pânico
}

\author{
Acute Pain Perception in Panic Disorder Patients
}

\author{
Fernando Pereira Miranda ${ }^{a}$, Ana Maria Crepaldi ${ }^{a}$, Fabrício Fernandes Almeida $^{a}$, \\ Elie Cheniaux ${ }^{c, d}$, Antonio Egídio Nardi ${ }^{c}$, J. Landeira-Fernandez ${ }^{b, e}$, \\ Antonio Pedro de Mello Cruz ${ }^{a} \&$ Wânia Cristina de Souza ${ }^{*}, a$ \\ ${ }^{a}$ Universidade de Brasilia, Brasília, Brasil, \\ ${ }^{b}$ Pontifícia Universidade Católica do Rio de Janeiro, Rio de Janeiro, Brasil, \\ ${ }^{c}$ Universidade Federal do Rio de Janeiro, Rio de Janeiro, Brasil, \\ ${ }^{d}$ Universidade do Estado do Rio de Janeiro, Rio de Janeiro, Brasil \\ $\&{ }^{e}$ Universidade Estácio de Sá, Rio de Janeiro, Brasil
}

\begin{abstract}
Resumo
O presente trabalho investigou experimentalmente os limiares nociceptivos e a percepção subjetiva de dor no transtorno de pânico (TP). Trinta e sete pacientes com TP foram voluntariamente submetidos a um Teste Pressor ao Frio (TPF), em que uma de suas mãos era mergulhada em um banho termostatizado de água fria $\left(7^{\circ} \mathrm{C}\right)$ por um período máximo de três minutos. A latência de retirada da mão da água foi utilizada como um índice de dor aguda, enquanto a experiência subjetiva de dor foi avaliada por meio do Questionário McGill de Dor e de uma escala visual analógica. Os resultados indicaram latências similares de retirada da mão em comparação a 37 sujeitos-controle sadios, mas uma experiência subjetiva de dor significantemente maior nos pacientes com TP. Esse padrão de resultados não apenas confirma a utilidade do teste de pressor ao frio para a indução e estudo experimental da dor aguda em laboratório, mas também sugere uma importante associação entre dor e ansiedade.

Palavras-chave: Dor, Teste de Pressor ao Frio, Transtorno de Pânico, Questionário McGill de Dor.
\end{abstract}

\begin{abstract}
The present study experimentally investigated the nociceptive threshold and the subjective pain perception in panic disorder (PD). Thirty seven PD patients were voluntarily submitted to a cold pressor test $(\mathrm{CPT})$ in which one of their hands was dipped into a thermostatized cold water bath $\left(7^{\circ} \mathrm{C}\right)$ for a maximum period of three minutes. Acute pain experience was assessed by measuring the hand retrieval latency, whereas the subjective pain experience was evaluated through McGill Pain Questionnaire and a pain visual analog scale. As compared to 37 healthy control-subjects, results indicated similar hand retrieval latencies but a significantly higher subjective pain experience in PD patients. Such pattern of results not only indicates the usefulness of the cold pressor test to induce and experimentally study pain in laboratory settings, but also suggests an important anxiety-pain association.

Keywords: Pain, Cold Pressor Test, Panic Disorder, Mcgill Pain Questionnaire.
\end{abstract}

O transtorno de pânico é uma psicopatologia caracterizada por ataques recorrentes e inesperados de pânico, acompanhados por um estado de ansiedade que se estende por pelo menos um mês acerca da possibilidade de novos ataques e das possíveis implicações ou conseqüências desagradáveis decorrentes dos mesmos. O ataque de pânico é definido por terror ou medo intenso na presença de taquicardia, hiperventilação, pressão arterial elevada, asfixia, náusea, desconforto abdominal, tontura, dores no peito e sensações subjetivas de pavor e morte iminente

\footnotetext{
" Endereço para correspondência: Universidade de Brasília, Instituto de Psicologia, Asa Norte, Brasília, DF, Brasil, CEP 70910-900. E-mail: wcsouza@unb.br Os autores agradecem à Fundação de Empreendimentos Científicos e Tecnológicos (FINATEC, Brasília-DF) e ao Conselho Nacional de Desenvolvimento Científico e Tecnológico.
}

(Associação Americana de Psiquiatria, 2000/2002; ver também Roy-Byrne, Craske, \& Stein, 2006; Salum, Blaya, Blaya, \& Manfro, 2009).

Tais reações fisiológicas e comportamentais são bastante semelhantes àquelas observadas em homens e animais em situações de intenso perigo real e proximal, exceto pelo fato de que, pelo menos, no primeiro ataque de pânico em um indivíduo com transtorno do pânico, estas reações são deflagradas subitamente e na ausência de fontes reais de perigo. Da mesma forma, as sensações subjetivas de ansiedade presentes no transtorno de pânico e que levam o indivíduo a temer um novo ataque de pânico apresentam certo grau de similaridade à sensação difusa de ansiedade experimentada por um indivíduo diante de sinais vagos, mas potencialmente perigosos. Esse tipo de ansiedade recorrente e constante é tão marcante no transtorno de pânico que cerca de $2 / 3$ destes pacientes 
desenvolvem agorafobia e passam a evitar lugares onde é difícil sair ou encontrar ajuda (Langs et al., 2000; Van Balkon et al., 1997).

Desde os estudos originais de Klein e Fink (1962) demonstrando que os principais tratamentos farmacológicos para a ansiedade não melhoram os sintomas de pânico, chegando, até mesmo em alguns casos, a agravarem o quadro, a relação entre ansiedade e pânico tem sido altamente debatida. Enquanto algumas evidências clínicas e experimentais sustentam a idéia de que a ansiedade e o pânico fazem parte de um mesmo contínuo (Basoglu, Marks, \& Sengun, 1992; Bouton, Mineka, \& Barlow, 2001), outras parecem sugerir, a exemplo da polêmica teoria proposta por Deakin e Graeff (1991), uma relação oposta na qual o medo inibiria o pânico.

De grande interesse experimental e teórico foi a demonstração de que padrões muito semelhantes aos de um ataque espontâneo de pânico, acompanhados de dor difusa na face e no peito, poderiam ser obtidos a partir da estimulação elétrica da matéria cinzenta periaquedutal em humanos sadios (Nashold, Wilson, \& Slaughter, 1969), uma das principais estruturas cerebrais envolvidas na modulação de estados de medo e ansiedade. Por esta razão, esta estrutura vem sido implicada em alguns dos principais modelos fisiopatológicos do transtorno de pânico (e.g. Deakin \& Graeff, 1991; Del-Bem, \& Graeff, 2009; Jenk, Moreau, \& Martin, 1995).

Paralelamente, desde que Bolles e Fanselow (1980) demonstram que o medo é capaz de inibir momentaneamente a dor por meio de um mecanismo opióide endógeno (Melzack, 1975), a relação entre os sistemas motivacionais de medo e de dor tem sido confirmada e implicada na fisopatologia de alguns transtornos psiquiátricos (e.g. Brandão \& Castilho, 2001; Cruz \& Landeira-Fernandez, 2001; Grimes, Meagher, \& Rhudy, 2004; Harris, 1996; Hirsh, George, Bialosky, \& Robinson, 2008; Landeira-Fernandez \& Cruz, 2007).

Considerando o fato de que o medo inibe a dor e a sugestão proposta por Deakin e Graeff (1991) de que o medo inibe o pânico, qual seria o padrão de percepção de dor aguda em sujeitos acometidos com transtorno de pânico? Embora a experiência subjetiva de dor crônica já tenha sido amplamente estudada em diversos transtornos de ansiedade e depressão (para revisões, ver Arntz \& Jong, 1993; Fleet, Lavoiea, \& Beitmane, 2000; Mayou, 1997; Soares Filho, Valença, \& Nardi, 2007), curiosamente poucos estudos utilizaram métodos experimentais para investigar a percepção de dor aguda no transtorno de pânico e, mesmo assim, os resultados são aparentemente contraditórios (Lautenbacher, Spernal, Schreiber, \& Krieg, 1999; Schmidt \& Cook, 1999). O presente estudo teve como objetivo investigar, por meio de um procedimento experimental adaptado para esta situação, os limiares nociceptivos e a percepção subjetiva de dor aguda em pacientes com transtorno de pânico submetidos ao teste de pressor ao frio. Considerando o aspecto multidimensional da dor (Guimarães, 1999), foram utilizadas medidas diretas e indiretas de percepção de dor aguda.

\section{Método}

\section{Participantes}

Trinta e sete portadores de transtorno de pânico (11 do sexo masculino e 26 do sexo feminino) participaram do estudo. Eles foram voluntariamente convidados para o estudo após admissão para tratamento e diagnóstico confirmatório prévio por médicos psiquiatras no Instituto de Psiquiatria da Universidade Federal do Rio de Janeiro e em clínicas particulares de Brasília, Distrito Federal. De uma amostra inicial de 44 pacientes convidados, 7 se recusaram a participar do estudo, resultando na amostra final de 37 pacientes. A idade média foi de 43,4 anos $(D P=11,55)$.

Para comparações, formando um grupo controle, 37 participantes saudáveis (11 do sexo masculino e 26 do sexo feminino) também foram selecionados nas mesmas cidades e localidades em que foram convidados os pacientes. O recrutamento destes participantes foi realizado por pareamento, convidando-se estudantes e funcionários dos respectivos locais, e a idade média foi de 42,56 anos $(D P=12,65)$. Para cada participante com transtorno de pânico foi selecionado via abordagem visual direta um voluntário saudável de mesmo sexo e idade próxima, sendo então convidado para ser submetido ao procedimento experimental na mesma localidade.

A seleção dos 37 participantes com transtorno de pânico se deu por conveniência, utilizando-se como critério inicial de inclusão o fato de terem sido admitidos para tratamento específico de transtorno de pânico nos setores e clínicas de atendimento nas cidades e locais em que vinham sendo atendidos. Atendido este critério, cada participante foi adicionalmente submetido a uma entrevista confirmatória do quadro sintomático utilizando-se o Mini International Neuropsychiatric Interview (MINI, versão 5.0.0), validada para a língua portuguesa e uso no Brasil por Amorim (2000). Os 37 participantes que constituíram esta amostra tiveram o quadro sintomático confirmado pelo MINI. Destes, 33 (89,19\%) faziam uso há pelo menos 15 dias de tratamento farmacológico concomitante com diferentes antidepressivos da classe dos antidepressivos tricíclicos ou inibidores seletivos de recaptação de serotonina. Também com base no MINI foram identificados 14 participantes $(37,83 \%)$ com diagnóstico de transtorno de pânico com agorafobia (CID F40.01 - DSM IV 300.21) e 4 com sintomatologia sugestiva de depressão (CID F32.1 - DSM IV 296.3).

Os voluntários sadios também foram submetidos à entrevista estruturada com o MINI. Participantes com eventual quadro sintomático de transtorno de pânico foram excluídos da amostra. De uma amostra inicial de 39 participantes, dois $(0,05 \%)$ foram excluídos por 
apresentarem quadro sintomatológico compatível com diagnóstico de transtorno de pânico. Foram excluídos pacientes com outro diagnóstico psiquiátrico nos 37 participantes que constituíram essa amostra de sujeitos-controle.

Além deste critério de exclusão, foram também excluídos de ambas as amostras, com transtorno de pânico e voluntários sadios, aqueles com queixa de dores crônicas ou dores nos braços causadas por artrite, artrose ou quaisquer processos inflamatórios ou cortes na mão ou braço direito, ou que tivessem feito uso de qualquer medicamento analgésico ou antiinflamatório nos cinco dias anteriores ao teste.

Cada participante preencheu e assinou termo de consentimento livre e esclarecido, o qual lhe informava os procedimentos e finalidades da pesquisa. No caso de dúvida de compreensão de qualquer ponto, o experimentador fornecia informações e esclarecimentos adicionais. O termo de consentimento somente era assinado mediante a inexistência de qualquer dúvida do participante. Nenhum tipo de coerção para a participação no estudo foi permitido tanto por parte do experimentador quanto do participante. Todos os participantes confirmaram o entendimento da instrução marcando uma afirmativa positiva no termo de consentimento.

\section{Materiais e Procedimento}

Todo o procedimento foi previamente aprovado tanto pelo Comitê de Ética em Pesquisa da Faculdade de Ciências da Saúde, na Universidade de Brasília, quanto pelo Comitê de Ética em Pesquisa do Instituto de Psiquiatria (IPUB), na Universidade Federal do Rio de Janeiro.

Uma vez com o termo de consentimento livre e esclarecido assinado e os procedimentos gerais do experimento explicados, cada participante teve sua mão direita inspecionada pelo experimentador para averiguação da inexistência de qualquer ferimento e comparação com as informações prestadas quando do recrutamento voluntário. Foi também solicitado que retirassem jóias ou objetos da mão e braço direito.

Para a indução do estímulo nociceptivo foi utilizado um banho termostatizado (Ética, Brasil, Mod 8943) com cuba interna $(30 \times 25 \times 15 \mathrm{~cm})$ para acomodação de 8 litros de água e controle automatizado de temperatura. O aparelho permitia a regulagem automática de temperatura na faixa de 0 a $45^{\circ} \mathrm{C}$, com erro de $\pm 0,5^{\circ} \mathrm{C}$. A água foi resfriada à temperatura de $7^{\circ} \mathrm{C}$, onde cada participante teve sua mão mergulhada até a altura de seu antebraço.

Lateralmente ao banho termostatizado foi colocada uma cadeira que dispunha de altura regulável. O participante era instruído a sentar-se nela e ajustar a altura da cadeira de forma a garantir que sua mão, punho e parte do antebraço direito pudessem ser submersos na água sem que $o$ cotovelo fosse retirado do apoio da cadeira. Após esse ajuste, uma pequena marca com caneta foi feita na altura correspondente ao antebraço de cada participante em relação à posição da cadeira e do banho termostatizado.
Sob a instrução do experimentador cada participante mergulhou sua mão na cuba de água até a altura de marca da caneta em seu antebraço, com a mão aberta e sem tocar no fundo, e recebeu a instrução de que deveria permanecer com ela mergulhada até o momento em que suportasse. O participante poderia interromper o experimento a qualquer momento que desejasse. Uma latência máxima de três minutos foi estabelecida para a retirada da mão. Se após este intervalo de tempo o participante não tivesse retirado sua mão da água, o experimentar solicitava que o mesmo a retirasse naquele exato momento. A latência de retirada da mão foi feita com o auxílio de um cronômetro digital (Timex, modelo TI5G811).

Como índices psicométricos de avaliação da experiência de dor aguda foram utilizados os seguintes parâmetros: (1) latência, em segundos, de retirada da mão da cuba de água fria; (2) escala visual analógica na qual o participante sinalizava com um traço de caneta o ponto que melhor traduzia sua experiência dolorosa em uma linha reta de $10 \mathrm{~cm}$, onde uma extremidade indicava "nenhuma dor" e a outra indicava "a pior dor imaginável". Adicionalmente, cada participante foi instruído a responder uma versão resumida do Questionário McGill de Dor, proposto originalmente por Melzack (1975), e validado para o Brasil por Pimenta e Teixeira (1996). O questionário consistiu de 15 descritores de dor, organizados em grupos e relacionados aos componentes sensorial, afetivo-motivacional e global da dor. Com base nestes descritores, foi feita uma avaliação global de dor, utilizando-se seis variáveis categóricas que se estenderam de "nenhuma dor" até uma experiência de "dor martirizante".

Os resultados das latências de retirada da mão foram analisados estatisticamente com base no teste $t$ de Student, enquanto as demais comparações foram feitas utilizando-se o teste Qui-quadrado para amostras independentes. Foi adotado um nível de significância de $p<0,05$.

\section{Resultados}

Os participantes portadores do transtorno de pânico mantiveram a mão na água à temperatura de $7^{\circ} \mathrm{C}$ (latência para retirada) por uma média de 66,93 s $(D P=30,57)$, e os participantes do grupo voluntários sadios por um tempo médio de $61,34 \mathrm{~s}(D P=34,53)$ (Figura 1). No teste $t$ para amostras independentes, os limites de confiança de $95 \%$ indicaram que, se o teste fosse repetido, a diferença das médias populacionais não seria significativa $(p=0,756)$.

Analisando-se o período de latência por gênero, verificou-se que o período médio de latência do grupo controle de homens foi de $58,86 \mathrm{~s}(D P=29,79)$ e de $91,14 \mathrm{~s}$ para o grupo dos pacientes $(D P=38,29) ; p=0,105$ (Figura 1). No grupo de mulheres saudáveis, o período médio de latência foi de 62,14 $(D P=31,46)$, enquanto que no grupo das portadoras de pânico, foi de 59,23 ( $D P=30,22)$; $p=0,756$ (Figura 2). Não foram observadas diferenças significantes $(p \mathrm{~s}>0,05)$. 


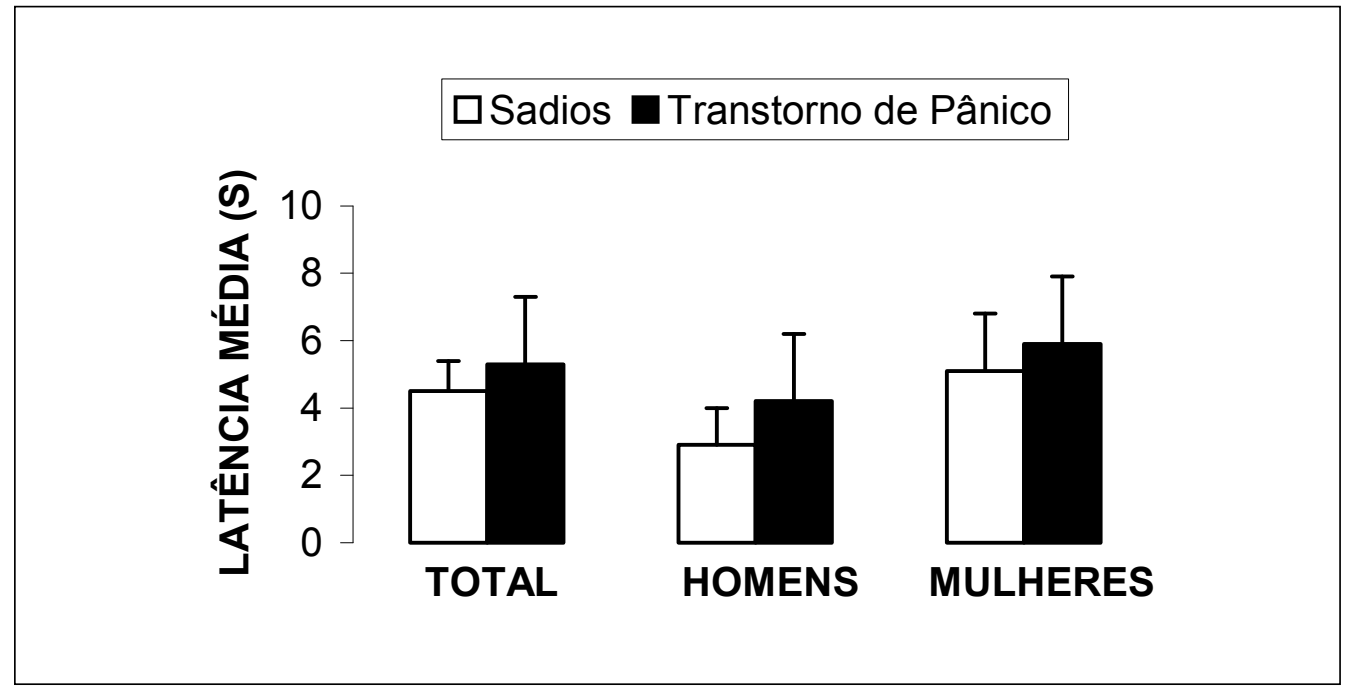

Figura 1. Média das latências de retirada da mão da água para os participantes sadios e com transtorno de pânico. Linhas verticais representam o erro padrão da média (EPM).

Os pacientes com transtorno de pânico mostraram uma tendência maior de experiência de dor na escala VAS $(M=5,30, D P=2,46)$ do que o grupo controle-controle de participantes $(M=4,51, D P=2,64)$. As diferenças, no entanto, não se mostraram estatisticamente significantes $(p=0,24)$ (Figura 2$)$. Houve também uma tendência de diferença de gênero, mas sem efeito esta- tístico significante. Entre os homens, o grupo controle apresentou a média de $2,93(D P=2,51)$, e o grupo de pacientes apresentou a média de $4,21(D P=1,36)$; $p=0,257$ (Figura 2). Entre as mulheres, foi observada uma média de 5,01 $(D P=2,53)$ para o grupo controle, e de $M=5,64(D P=2,65)$ para as mulheres portadoras de pânico; $p=0,424$ (Figura 2).

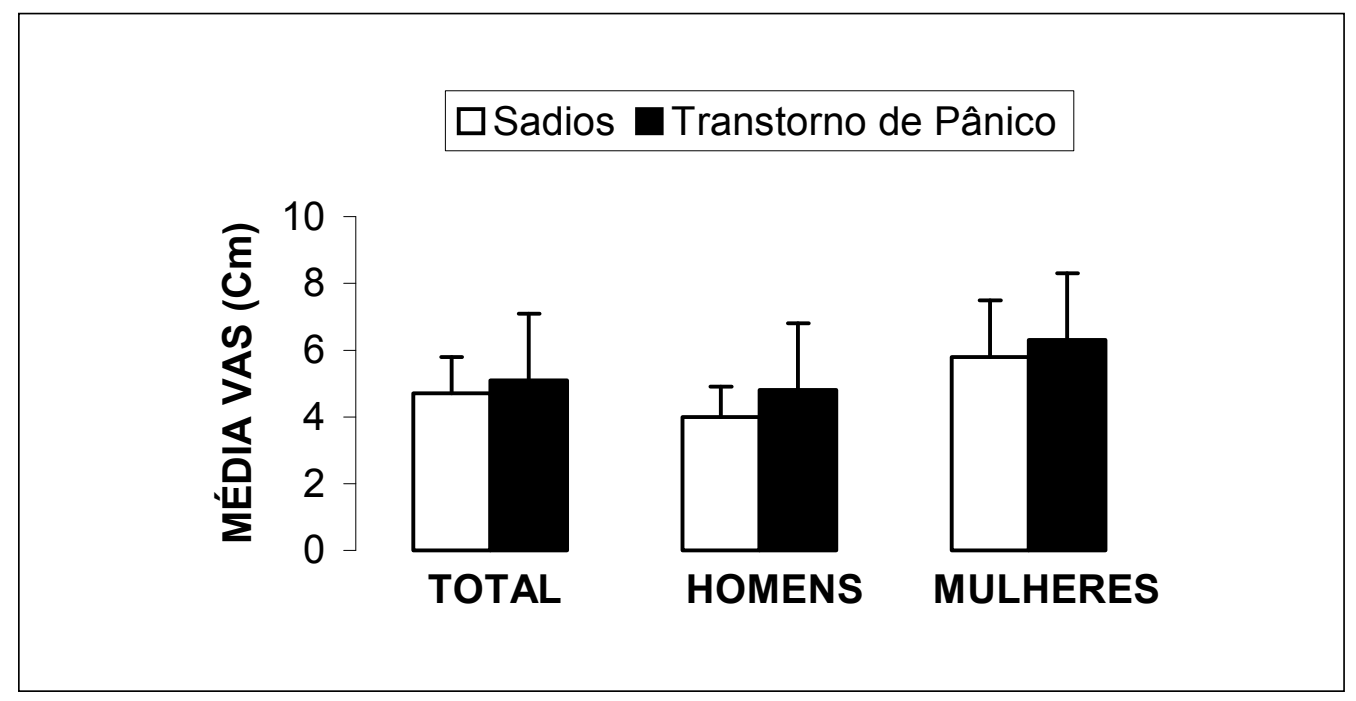

Figura 2. Valores médios em centímetros na escala visual analógica para os participantes sadios e com transtorno de pânico. Linhas verticais representam o erro padrão da média (EPM).

Por sua vez, quando os participantes relataram o nível de dor a partir da escala unidimensional que mediu a percepção global da dor no Questionário McGill, os pacientes com transtorno de pânico julgaram os descritores de dor significantemente mais aversivos $(x=2,89)$ (Qui-Qua- drado, $p$ s $<0,05)$ do que os voluntários sadios $(x=1,93)$. O $V$ de Cramer obtido foi de 0,42 , indicando que aproximadamente $17,6 \%$ das variações de freqüências de dor foram explicadas pelas variações de freqüências nos grupos utilizados no experimento. 
Miranda, F. P., Crepaldi, A. M., Almeida, F. F., Cheniaux, E., Nardi, A. E., Landeira-Fernandez, J., Cruz, A. P. M. \& Souza, W. C. (2011). Percepção de Dor Aguda em Pacientes com Transtorno de Pânico.

Tabela 1

Freqüências dos Descritores de Dor da Forma Reduzida do Questionário Mcgill de Dor dos Participantes com Transtorno de Pânico e Voluntários Sadios

\begin{tabular}{llccccccc}
\hline & & \multicolumn{7}{c}{ Descritores de Dor } \\
\cline { 3 - 7 } Grupo & Frequência & Nenhuma & Branda & Desconfortável & Aflitiva & Horrível & Martirizante & Total \\
\hline Controle & Observado & 10 & 17 & 45 & 24 & 3 & 0 & 99 \\
& Esperado & 5 & 12 & 43 & 24 & 6,5 & 8,5 & 99 \\
Transtorno & Observado & 0 & 7 & 41 & 24 & $10^{*}$ & $17 *$ & 99 \\
de Pânico & Esperado & 5 & 12 & 43 & 24 & 6,5 & 8,5 & 99 \\
Total & Observado & 10 & 24 & 86 & 48 & 13 & 17 & 198 \\
& Esperado & 10 & 24 & 86 & 48 & 13 & 17 & 198 \\
\hline
\end{tabular}

* Indica diferença estatisticamente significante a um nível de $p<0,05$.

\section{Discussão}

O principal objetivo deste estudo foi investigar a percepção de dor aguda em pacientes com transtorno de pânico por meio de um procedimento experimental baseado em um teste amplamente utilizado para o estudo da dor, o teste pressor ao frio. Paralelamente, considerando o aspecto multifatorial da dor, procurou-se também utilizar medidas que incluíram desde testes psicométricos até medidas de auto-relato.

A percepção de dor aguda detectada pela latência de retirada da mão da água fria e pela escala visual analógica mostraram padrões semelhantes nos portadores de transtorno de pânico em relação aos voluntários sadios. Se, por um lado, os resultados dessas medidas psicométricas corroboram estudos prévios onde a percepção de dor aguda também se mostrou inalterada no transtorno de pânico (Lautenbacher et al, 1999; Roy-Byrne et al., 2006), por outro lado contrastam com o estudo de Schmidt e Cook (1999). Por meio de uma escala visual analógica e de medidas de auto-relato, este autores observaram uma percepção maior de dor aguda em pacientes com transtorno de pânico em resposta ao teste de pressor ao frio.

As razões dessas discrepâncias são desconhecidas, mas podem envolver desde seleção da amostra, gênero, comorbidades ou não com outros transtornos psiquiátricos, até variáveis metodológicas como as medidas de avaliação de dor e características dos estímulos nociceptivos empregados. Por exemplo, embora o teste de pressor ao frio seja amplamente utilizado e considerado fidedigno (para revisão, ver Brodie, MacDonald, Mitchell, \& Raymond, 2004) para o estudo da dor, ele é bastante variado em termos de procedimento e aparentemente dependente da temperatura utilizada. No estudo de Schmidt e Cook (1999), ao contrário do presente estudo e dos procedimentos empregados por Lautenbacher et al. (1999) e Roy-Byrne et al. (2006), o estímulo nociceptivo foi produzido por uma compressa imersa em produto químico cujo contato com o pescoço do participante causava a sensação térmica de frio. De fato, os autores sequer tiveram controle sobre a temperatura real produzida. Talvez a temperatura de $7^{\circ} \mathrm{C}$ que utilizamos não tenha sido percebida como suficientemente aversiva, haja vista a porcentagem de $13,51 \%$ dos participantes com transtorno de pânico e $21,62 \%$ dos voluntários sadios alcançarem o teto de três minutos de permanência da mão na água. Tal fato é indicativo de que provavelmente o limiar de dor dos participantes possa não ter sido atingido com a temperatura proposta no experimento. Estudos recentes de nosso próprio laboratório (dados não publicados) sugerem que a temperatura de $3^{\circ} \mathrm{C}$ é mais adequada para este tipo de procedimento.

Embora as medidas de latência para retirada e os escores descritos pela escala visual analógica não tenham sido significantemente diferentes entre os grupos, maiores níveis de dor foram relatados pelos pacientes portadores do transtorno de pânico através da medida de avaliação global de dor do Questionário McGill. A experiência subjetiva de dor foi descrita por esses pacientes como "horrível" e "martirizante". Fleet, Dupuis, Marchand, Burelle e Beitman (1997) encontram resultados semelhantes também utilizando uma forma resumida do Questionário McGill. Pelo menos em parte, este padrão de resultados sugere as medidas de auto-relato são mais sensíveis para detectar a experiência de dor aguda do que outras medidas psicométricas.

As previsões baseadas tanto no modelo de Bolles e Fanselow (1980), cujo medo inibe a dor, quanto no modelo de Deakin e Graeff (1991), cujo medo inibe o pânico, não foram confirmadas. Ao contrário, as diferenças encontradas nas medidas de auto-relato mostraram uma percepção subjetiva de dor aguda maior nos pacientes com transtorno de pânico. Este padrão para dor aguda é semelhante à alta correlação positiva observada entre dor crônica e transtornos de ansiedade (Asmundson, Kuperos, \& Norton, 1997). Indivíduos com dor crônica tendem a 
ser mais ansiosos que populações de indivíduos sadios e pacientes com transtorno de pânico frequentemente relatam dor persistente (Meagher, \& Rhudy, 2000; Schmidt \& Telch, 1997; Telch, Ilai, Valentiner, \& Craske, 1994).

No entanto, cabe ressaltar, conforme demonstrado recentemente por Chen, Tsuchiya, Kawakami e Furukawa (2009), que cerca de $30 \%$ dos ataques de pânico ocorrem na ausência de uma experiência subjetiva de medo e, mesmos nesses casos, os demais componentes característicos de sensação de dor no peito e abdômen estão presentes. Nossos participantes foram submetidos à estimulação aversiva em uma condição de descanso e os portadores de transtorno de pânico estavam em condições assintomáticas no instante do teste. Se o mesmo procedimento tivesse sido adotado após um ataque de pânico, é possível que a resposta de analgesia se mostrasse diferente.

Acreditamos que investigações futuras possam contornar algumas limitações do presente trabalho. Estudos que venham a ser realizados com pacientes com manifestação de ataques iniciais de pânico e em admissão para tratamento, ainda sem medicação, embora complexo, seria viável em um período maior. Esse procedimento minimizaria possíveis efeitos influenciadores da medicação sobre os limiares de dor experimental. Tais estudos poderiam ainda replicar o método com uma grande amostra, visto que a do presente estudo é pequena, e com participantes com perfis socioeconômicos e faixas etárias variadas.

Finalmente, nossos resultados como um todo não apenas confirmam a utilidade do teste de pressor ao frio para a indução e estudo experimental da dor aguda em condições laboratoriais, mas também apontam uma importante associação entre dor e ansiedade. No entanto, estudos futuros são necessários para um melhor entendimento se esse tipo de ansiedade no pânico é acompanhado por uma maior ou menor percepção de dor aguda.

\section{Referências}

Amorim, P. (2000). Mini International Neuropsychiatric Interview (MINI): Validação de entrevista breve para diagnóstico de transtornos mentais. Revista Brasileira de Psiquiatria, 22(3), 106-115.

Arntz, A., \& Jong, P. D. (1993). Anxiety, attention and pain. Journal of Psychosomatic Research, 37, 423-432.

Asmundson, G. J., Kuperos, J. L., \& Norton, G. R. (1997). Do patients with chronic pain selectively attend to pain-related information? Preliminary evidence for the mediating role of fear. Pain, 72(1), 27-33.

Associação Americana de Psiquiatria. (2002). DSM-IV (TR), Manual Diagnóstico e Estatístico de Transtornos Mentais (4 ed. rev.). Porto Alegre, RS: Artmed. (Original work published 2000)

Basoglu, M., Marks, I. M., \& Sengun, S. A. (1992). Prospective study of panic and anxiety in agoraphobia with panic disorder. British Journal of Psychiatry, 160, 57-64.
Bolles, R. C., \& Fanselow, M. S. (1980). A perceptual-defensiverecuperative model of fear and pain. Behavioral and Brain Science, 3(2), 291-323.

Bouton, M. E., Mineka, S., \& Barlow, D. H. (2001). A modern learning theory perspective on the etiology of panic disorder. Psychological Review, 108, 4-32.

Brandão, M. L., \& Castilho, V. M. (2001). Conditioned antinociception and freezing using electrical stimulation of the dorsal periaqueductal gray or inferior colliculus as unconditioned stimulus are differentially regulated by 5HT2A receptors in rats. Psychopharmacology, 155(2), 154162.

Brodie, E. E., MacDonald, A. R., Mitchell, L. A., \& Raymond, A. (2004). Temperature and the Cold Pressor Test. The Journal of Pain, 5, 233-238.

Chen, J., Tsuchiya, Kawakami, N., \& Furukawa, T. S. (2009). Non-fearful vs. fearful panic attacks: A general population study from the National Comorbidity Survey. Journal of Affective Disorders, 112, 273-278.

Cruz, A. P. M., \& Landeira-Fernandez, J. (2001). A ciência do medo e da dor. Ciência Hoje, 174, 16-22.

Deakin, J. F. W., \& Graeff, F. G. (1991). 5-HT and mechanisms of defense. Journal of Psychofarmacology, 5(4), 305-315.

Del-Bem, C. M., \& Graeff, F. G. (2009). Panic Disorder: Is the PAG involved? Neural Plasticity, 1-9.

Fleet, R. P., Dupuis, G., Marchand, A., Burelle, D., \& Beitman, B. D. (1997). Detecting panic disorder in emergency department chest pain patients: A validated model to improve recognition. Annals of Behavioral Medicine, 19, 124-131.

Fleet, R. P., Lavoiea, K., \& Beitmane, B. D. (2000). Is panic disorder associated with coronary artery disease? A critical review of the literature. Journal of Psychosomatic Research, 48, 347-356.

Grimes, J. S., Meagher, M. W., \& Rhudy, J. L. (2004). Fearinduced hypoalgesia in humans: Effects on low intensity thermal stimulation and finger temperature. Journal of Pain, 5(8), 458-68.

Guimarães, S. S. (1999). Introdução ao estudo da dor. In M. M. J. Carvalho (Ed.), Dor, um estudo multidisciplinar (pp. 1430). São Paulo, SP: Summus.

Harris, J. A. (1996). Descending antinociceptive mechanisms in the brain-stem: Their role in the animal's defensive system. Journal of Physiology, 90, 15-25.

Hirsh, A. T, George, S. Z., Bialosky, J. E., \& Robinson, M. E. (2008). Fear of pain, pain catastrophizing, and acute pain perception: Relative prediction and timing of assessment. The Journal of Pain, 9(9), 806-812.

Jenk, F., Moreau, J. L., \& Martin, J. R. (1995). Dorsal periaqueductal gray-induced aversion as a stimulation of anxiety: Elements of face and predictive validity. Psychiatry Research, 57, 181-191.

Klein, D. F., \& Fink, M. (1962). Psychiatric reactions patterns to imipramine. American Journal of Psychiatry, 119, 432438.

Landeira-Fernandez, J., \& Cruz, A. P. M. (2007). Medo e dor e a origem da ansiedade e do pânico. In J. Landeira-Fernandez \& M. T. A. Silva, Intersecções entre psicologia e neurociências (pp. 217-239). Rio de Janeiro, RJ: MedBook.

Langs, G., Quehenberger, F., Fabisch, K., Klug, G., Fabisch, H., \& Zapotoczky, H. G. (2000). The development of agoraphobia in panic disorder: A predictive processes? Journal of Affective Disorders, 58, 43-50. 
Miranda, F. P., Crepaldi, A. M., Almeida, F. F., Cheniaux, E., Nardi, A. E., Landeira-Fernandez, J., Cruz, A. P. M. \& Souza, W. C. (2011).

Percepção de Dor Aguda em Pacientes com Transtorno de Pânico.

Lautenbacher, S., Spernal, J., Schreiber, W., \& Krieg, J.-C. (1999). Relationship between clinical pain complaints and pain sensitivity in patients with depression and panic disorder. Psychosomatic Medicine, 61, 822-827.

Mayou, R. (1997). Chest pain, palpitations and panic. Journal of Psychosomatic Research, 44(1), 53-70.

Meagher, M. W., \& Rhudy, J. L. (2000). Fear and anxiety: Divergent effects on human pain thresholds. Pain, 84(1), 65-75.

Melzack, R. (1975). The Mcgill Pain Questionaire: Major properties and scoring methods. Pain, 1, 277-299.

Nashold, B. S., Jr., Wilson, W. P., \& Slaugther, D. G. (1969). Sensations evoked by stimulation in the midbrain of man. Journal of Neurosurgery, 30(1), 14-24.

Pimenta, C. A. M., \& Teixeira, M. J. (1996). Questionário de dor McGill: Proposta de adaptação para a língua portuguesa. Revista Escola de Enfermagem - USP, 30(3), 473-83.

Roy-Byrne, P. P., Craske, M. G., \& Stein, M. B. (2006). Panic disorder. Lancet, 368(9540), 1023-1032.

Salum, G. A., Blaya, C. I., Blaya, C., \& Manfro, G. G. (2009). Transtorno de pânico. Revista de Psiquiatria do Rio Grande do Sul, 31(2), 86-94.

Schmidt, N. B., \& Cook, J. (1999). Effects of anxiety sensitivity on anxiety and pain during a cold pressor challenge in patients with panic disorder. Behaviour Research and Therapy, 37, 313-323.

Schmidt, N. B., \& Telch, M. J. (1997). Nonpsychiatric medical comorbidity, health perceptions, and treatment outcome in patients with panic disorder. Health Psychology, 16(2), 114122.

Soares Filho, G. L. F., Valença, A. M., \& Nardi, A. E. (2007). Dor torácica no transtorno de pânico: Sintoma somático ou manifestação de doença arterial coronariana? Revista de Psiquiatria Clínica, 34(2), 97-102.

Telch, M. J., Ilai, D., Valentiner, D., \& Craske, M. G. (1994). Mismatch of fear, panic and performance. Behaviour Research and Therapy, 32, 691-700.

Van Balkon, A., Bakker, A., Spinhoven, P., Blaauw, B., Smeenk, S., \& Ruesink, B. (1997). A meta-analysis of the treatment of panic disorder with or without agoraphobia: A comparison of psychopharmacological, cognitive-behavioral, and combination treatments. The Journal of Nervous \& Mental Disease, 185(8), 510-516. 